\title{
A Poor Outcome after Inappropriate Use of an Over-the-Counter Fetal Listening Device
}

Michael W. Kalsman, MD

Home monitoring devices have been available for years, as far back as the introduction of the thermometer in the late 1800s. As technology has advanced, a large number of home monitoring devices and diagnostic tests have become available to the general public. The Food and Drug Administration (FDA) oversees this industry and has established two primary criteria for approval of such items: ease of use and the ability to safely and effectively interpret the results.

Recently, listening devices have become available that allow for auscultation of fetal heart tones during pregnancy. These devices are not deemed medical devices and hence do not fall under regulatory constraints of the FDA. However, as with herbal products and other nonregulated devices, inappropriate usage has the potential for harm.

\section{Case Report}

A 26-year-old woman (gravida 2, para 1) presented to labor and delivery at 39.5 weeks of gestation with the report of no fetal movement for 2 days. She stated that she had not called sooner because she was using a home fetal listening device 2 to 3 times a day and was hearing the baby's heartbeat. She presented only after having difficulty finding the heart beat. She denied any symptoms of labor, infection, or preeclampsia. Her pregnancy had been without any complications or concerns. Her past obstetrical history was only significant for induction at term for preeclampsia, but without any adverse outcomes. She was healthy with no significant past medical history.

Initial evaluation revealed normal vital signs and a normal physical examination. The fetal heart rate was noted to be in the range of 150 beats/min, with decreased variability and occasional mild variable

Submitted, revised, 28 March 2003.

From the Department of Family Medicine, State University New York-Buffalo, Olean, NY. Address correspondence to Michael Kalsman, MD, SUNY-Buffalo Rural Family Practice Residency, University Primary Care, 500 Main St., Olean, NY 14760 (e-mail: mkalsman@acsu.buffalo.edu). decelerations. After approximately 40 minutes of a nonreactive tracing, a biophysical profile was performed, scoring 2/10 ( +2 for fluid). Given the troubling biophysical profile, the patient consented to and was taken for immediate cesarean delivery. She delivered a 3.34-kg male baby with Apgar scores of $8 / 8$ at 1 and 5 minutes. The cord $\mathrm{pH}$ drawn at delivery was 7.20 , with a base excess of -10.0 . The initial newborn examination was significant for a labored respiratory rate of 44 , oxygen saturation of $79 \%$ on room air, and decreased muscle tone. The newborn was placed under an oxygen hood, and an infusion of concentrated dextrose (D10; $100 \mathrm{~g} / \mathrm{L})$ was needed to maintain appropriate blood sugars. Despite an improving clinical picture, at 19 hours of life, the newborn had a seizure lasting 2 to 3 minutes that ceased spontaneously. Laboratory studies were performed; the results were notable for a slightly low sodium and chloride at 128 and $94 \mathrm{mmol} / \mathrm{L}$, respectively. The white blood cell count was $17,300 / \mu \mathrm{L}$ with a normal differential. Blood cultures were drawn, and the newborn was started on intravenous antibiotics. Approximately 45 minutes after the initial seizure, there was a second seizure, at which time phenobarbital was given and arrangements were made for transfer to the tertiary care center.

The newborn did well over the next 5 days without any further seizures. A full evaluation was performed that revealed a grade 1 intracranial hemorrhage. All cultures were negative, and there was no evidence of congenital infections. Cardiac evaluation was also normal. The final diagnosis given was ischemic brain injury with seizures. At 6 months of age, the infant demonstrated slight right-sided hemiplegia but otherwise had normal growth and development.

\section{Discussion}

This case is reported to demonstrate that anyone can misuse over-the-counter monitoring devices and that misuse may lead to potential harm. The patient is a graduate student who consistently dem- 
onstrates interest in every aspect of her prenatal care. She frequently asks questions and demands explanations. Yet when asked why she did not contact the physician sooner, she simply stated, "I was lulled into this false sense of security when I heard my baby's heartbeat."

Patient compliance with nonregulated over-thecounter (OTC) devices is not often studied. An extensive literature search found no published studies on the topic. If we assume that compliance is similar to that for OTC medications, there are data suggesting that compliance with product labeling is less than desirable and can lead to harm. ${ }^{1,2}$ Recently, the FDA released reports about unintentional acetaminophen toxicity. ${ }^{3}$ The FDA states that noncompliance with product labeling is the leading cause of harm. They are looking specifically at consumer usage patterns and labeling revisions. In the current case, we reviewed the product labeling on the specific product that the patient used. There was a disclaimer in small print at the bottom of the package back. The disclaimer was again noted on page 12 of 14 within the instruction manual. The disclaimer emphasized that it was not a medical device and that it should not be used as a substitute for prenatal care. The instructions also state: "It's quite normal that there will be times you will not be able to hear your baby's sounds." When the patient was asked if she recalled any such warnings, she stated that she had read all the instructions and had no recollection of such warnings. Given what we do know about patient compliance, it is not unreasonable to assume that if this educated and conscientious mother had a bad outcome, patients with less insight or education may have similar or worse outcomes. As the price of these devices decrease (currently $\$ 30$ to 50 ), they will become more available, thus increasing the likelihood of an event.

Because of the unclear cause of the intrauterine hypoxia, it is possible that this outcome was unavoidable. The intention of this report is to demonstrate inappropriate usage of OTC listening devices. A literature review from 1966 to present found no published articles on this topic. There are approximately 18 reports on home antenatal testing. The majority of these involve the use of telemetry-based monitoring in high-risk populations. The only type of self-interpreted home monitoring that has been advocated or studied is fetal kick counts. Two studies were found looking at outcomes in patients with decreased fetal movement. ${ }^{4,5}$ Neither study found a significant difference in neonatal outcome; however the study by Ahn et $\mathrm{al}^{4}$ found a higher incidence of oligohydramnios with decreased fetal movement. This study also found that reassuring non-stress test and amniotic fluid assessment in patients with decreased fetal movement correlated with lower rates of cesarean delivery for fetal distress and Apgar scores less than 7. Studies looking a home fetal movement counting have been done and have failed to show any improved outcomes in low risk populations. ${ }^{6}$ Nonetheless, it is easy to hypothesize that earlier care might have prevented this degree of neonatal injury, and continued delay may have worsened the outcome.

\section{Conclusion}

As with herbal medications and other nonregulated devices, home fetal-listening devices have potential for harm. More research is needed to precisely define the extent of harm. However, prenatal care providers need to recognize this issue and include it as a part of routine prenatal screening and education. In addition, consumer product advocacy is needed to ensure proper labeling and warnings.

\section{References}

1. Hughes CM, McElnay JC, Fleming GF. Benefits and risks of self medication. Drug Saf 2001;24: 1027-3.

2. Hallas J, Jensen KB, Grodum E, Damsbo N, Gram LF. Drug-related admissions to a department of medical gastroenterology. The role of self-medicated and prescribed drugs. Scand J Gastroenterol 1991;26:174-8.

3. Unintentional acetaminophen hepatotoxicity. Washington DC: Nonprescription Drugs Advisory Committee, Food and Drug Administration; 2002. Available at: URL: http://www.fda.gov/ohrms/dockets/ ac/02/transcripts/3882T1.htm

4. Ahn MO, Phelan JP, Smith CV, Jacobs N, Rutherford SE. Antepartum fetal surveillance in the patient with decreased fetal movement. Am J Obstet Gynecol 1987;157:860-4.

5. Harrington K, Thompson O, Jordan L, Page J, Carpenter RG, Campbell S. Obstetric outcome in women who present with a reduction in fetal movements in the third trimester of pregnancy. J Perinat Med 1998;26:77-8.

6. Grant A, Elbourne D, Valentin L, Alexander A. Routine formal fetal movement counting and risk of antepartum late death in normally formed singletons. Lancet 1989;2:345-34. 